\title{
MafA is a dedicated activator of the insulin gene in vivo
}

\author{
Isabella Artner ${ }^{1}$, Yan Hang ${ }^{1}$, Min Guo ${ }^{1}$, Guoqiang Gu $^{2}$ and Roland Stein ${ }^{1,2}$ \\ Departments of ${ }^{1}$ Molecular Physiology and Biophysics and ${ }^{2}$ Cell and Developmental Biology, Vanderbilt University Medical Center, Nashville, \\ Tennessee 37205, USA \\ (Correspondence should be addressed to I Artner who is now at Developmental Biology Program, Stem Cell Center, Lund University, Lund 22184, Sweden; \\ Email: isabella.artner@med.lu.se)
}

\begin{abstract}
As successful generation of insulin-producing cells could be used for diabetes treatment, a concerted effort is being made to understand the molecular programs underlying islet $\beta$-cell formation and function. The closely related MafA and MafB transcription factors are both key mammalian $\beta$-cell regulators. MafA and MafB are co-expressed in insulin ${ }^{+} \beta$-cells during embryogenesis, while in the adult pancreas only MafA is produced in $\beta$-cells and MafB in glucagon ${ }^{+} \alpha$-cells. MafB ${ }^{-1-}$ animals are also deficient in insulin ${ }^{+}$and glucagon ${ }^{+}$cell production during embryogenesis. However, only MafA overexpression selectively induced endogenous Insulin mRNA production in cell line-based assays, while MafB specifically promoted Glucagon expression. Here, we analyzed whether these factors were sufficient to induce insulin ${ }^{+}$and/or glucagon $^{+}$cell formation within embryonic endoderm
\end{abstract}

using the chick in ovo electroporation assay. Ectopic expression of MafA, but not MafB, promoted Insulin production; however, neither MafA nor MafB were capable of inducing Glucagon. Co-electroporation of MafA with the Ngn3 transcription factor resulted in the development of more organized cell clusters containing both insulin- and glucagonproducing cells. Analysis of chimeric proteins of MafA and MafB demonstrated that chick Insulin activation depended on sequences within the MafA C-terminal DNA-binding domain. MafA was also bound to Insulin and Glucagon transcriptional control sequences in mouse embryonic pancreas and $\beta$-cell lines. Collectively, these results demonstrate a unique ability for MafA to independently activate Insulin transcription.

Journal of Endocrinology (2008) 198, 271-279

\section{Introduction}

Encouraging results obtained in treating type 1 diabetes by islet transplantation have stimulated efforts to develop an abundant source of functional insulin-producing cells (Shapiro et al. 2000). Islet-enriched transcription factors have been shown to play fundamental roles in pancreas development and function, especially those linked to endocrine cell-specific expression of the hormones glucagon and insulin (reviewed by Sander \& German 1997, Servitja \& Ferrer 2004). Expression of these hormones is crucial for maintaining glucose homeostasis, as glucagon released from $\boldsymbol{\alpha}$-cells stimulates the mobilization of glucose through gluconeogenesis and glycogenolysis to prevent hypoglycemia, while insulin released from $\beta$-cells promotes glucose storage. Physiological glucose levels are maintained through the counter-regulatory actions of these hormones in peripheral tissues, with defects in $\alpha$ - and $\beta$-cell activity resulting in the inability of diabetics to maintain normoglycemia (Del Prato \& Marchetti 2004).

A key role in both islet cell development and function has been established for many transcriptional regulators of the Insulin and Glucagon genes. Transcription of these hormone genes is controlled by a number of factors, some shared, that bind to $5^{\prime}$ cis-regulatory elements in their enhancer region (e.g. Insulin: Pax6, Pdx1, NeuroD1 (also referred to as $\beta 2$ ), MafA (reviewed in Melloul et al. (2002), Artner \& Stein (2008)); Glucagon: Pax6 (Sander et al. 1997, Ritz-Laser et al. 1999), NeuroD1 (Naya et al. 1995), MafB (Artner et al. 2006, Nishimura et al. 2006), Brn4 (Hussain et al. 2002). Islet $\alpha$ - and $\beta$-cell development is impaired in Pax 6 mutant mice (Sander et al. 1997, St-Onge et al. 1997, Ashery-Padan et al. 2004), while the loss of NeuroD1 results in a severe, but general reduction in islet endocrine cells (Naya et al. 1997). Pdx1 acts upstream of Pax6 and NeuroD1, as knockout animals are apancreatic due to defects in both endocrine and exocrine cell differentiation (Jonsson et al. 1994, Ahlgren et al. 1996, Offield et al. 1996). Pdx1, NeuroD1, and MafA are also critical in mediating Insulin's response to glucose, the most important effector of $\beta$-cell function. In addition, dysfunctional heterozygous mutations in PDX1 (Stoffers et al. 1997), NEUROD1 (Malecki et al. 1999), and PAX6 (Dumonteil et al. 1998) cause diabetes in humans, presumably due to reduced expression of target genes required for proper islet cell function.

MafA and MafB were only recently linked to Insulin and Glucagon expression and represent the principal members of the large Maf transcription factor family expressed in the 
pancreas (Olbrot et al. 2002, Matsuoka et al. 2003, Artner et al. 2006, Nishimura et al. 2006). MafA is initially detected in insulin-producing cells at embryonic day (E)13.5 in mice, coinciding with the onset of mature $\beta$-cell differentiation (Matsuoka et al. 2004). By contrast, MafB is detected in both early (i.e. $<$ E13.5) and late $\left(>\right.$ E13.5) phase insulin ${ }^{+}$and glucagon $^{+}$cells during development (Artner et al. 2006). MafB expression becomes restricted to islet $\alpha$-cells soon after birth (Artner et al. 2006, Nishimura et al. 2006), while MafA (Matsuoka et al. 2004) is always only in $\beta$-cells. The expression of MafA and MafB primarily in hormoneproducing cells, and not in endocrine precursor cells, is very unusual when compared with other pancreas-enriched transcription factors. These findings indicated that MafA and MafB were both critical to $\beta$-cell formation. However, only $\mathrm{MafB}^{-1-}$ animals are deficient in insulin ${ }^{+}$and glucagon ${ }^{+}$ cell production during embryogenesis (Artner et al. 2007, Nishimura et al. 2008), although Maf $A^{-/-}$mice are diabetic due to defects in adult glucose sensing and insulin secretion capacity (Zhang et al. 2005).

Ectopic expression of islet-enriched transcriptional regulators in chick embryonic endoderm has been employed to assess their independent ability to induce endocrine cell differentiation. Previous experiments demonstrated that only Ngn3 and Myt1 were capable of inducing the formation of glucagon- and somatostatin-producing cells, while many other regulators of hormone gene expression and/or pancreas cell development had no effect (Grapin-Botton et al. 2001, Gu et al. 2004). Here, we examined whether newly characterized MafA and MafB were capable of activating hormone gene expression in this in ovo assay. MafA induced the formation of insulin-producing cells in embryonic gut endoderm and co-expression of MafA with Ngn3 resulted in the formation of islet-like clusters of insulin ${ }^{+}$and glucagon ${ }^{+}$cells. The effect of MafA on insulin production did not appear to be mediated by other islet-enriched transcription factors, including Pdx1, Nkx2.2, Pax6, or Is11. By contrast, MafB expression did not result in the production of hormone ${ }^{+}$ cells. Analysis of MafA/MafB chimeric proteins indicated a special significance for the C-terminal DNA-binding domain of MafA in endogenous chick Insulin activation, although Insulin and Glucagon control sequences bound both MafA and MafB in developing $\alpha$ - and $\beta$-cells. Collectively, these results demonstrate a fundamental difference between closely related MafA and MafB in Insulin gene transcription, presumably reflecting their ability to recruit factors essential in cooperative activation.

\section{Materials and Methods}

\section{ChIP assay}

Staged embryos were obtained from Balb/c mice. The day of vaginal plug discovery was designated E0.5. The animal studies were approved by the Vanderbilt University Animal
Care and Use Committee in accordance with current federal regulations. E18.5 pancreata, $\beta$ TC3 cells, and aTC6 cells were formaldehyde cross-linked, and the sonicated proteinDNA complexes isolated under conditions described previously (Matsuoka et al. 2003, Phuc Le et al. 2005). Sonicated chromatin was incubated $12-14 \mathrm{~h}$ at $4{ }^{\circ} \mathrm{C}$ with rabbit $\alpha$-MafA (Bethyl Laboratories, Montgomery, TX, USA) or $\alpha$-MafB (Bethyl Laboratories) and the complexes isolated with A/G-agarose (Santa Cruz Biotechnology, Santa Cruz, CA, USA). PCR was performed on one-tenth of the purified immunoprecipitated DNA using Readyto-Go PCR beads (Amersham Pharmacia Biotech) and 15 pmol of the Insulin $\left({ }^{-378}\right.$ GGAACTGTGAAACAGTCCAAGG and ${ }^{-46}$ CCCCCTGGACTTTGCTGTTTG), Glucagon $\left({ }^{-353}\right.$ CCAAATCAAGGGATAAGACCCTC and ${ }^{+7}$ AAGCTCTGCCCTTCTGCACCAG), and phosphoenolpyruvate carboxykinase (PEPCK) $\left({ }^{-434}\right.$ GAGTGACACCTCACAGCTGTGG and ${ }^{-96}$ GGCAGGCCTTTGGATCATAGCC) transcriptional control region primers. The amplified products were resolved on a $1.4 \%$ agarose gel in Tris-acetate-EDTA buffer containing ethidium bromide.

\section{In ovo electroporation}

In ovo electroporation was performed as described previously (Grapin-Botton et al. 2001, Xu et al. 2006). Embryos were electroporated with cytomegalovirus (CMV) enhancerdriven pCIG vectors (Megason \& McMahon 2002, Dasen et al. 2003) of mouse MafA, MafB, chimeric MafA/MafB, Ngn3, and/or Pdx1 at Hamburger \& Hamilton (1951) (HH) stages 11-13. Plasmids were injected at a final concentration of $2 \mu \mathrm{g} / \mu \mathrm{l}$, which resulted in in ovo expression of proteins within physiological levels (Dasen et al. 2003). Three pulses $(50 \mathrm{~ms}, 17 \mathrm{~V})$ were applied to the embryo using a BTX 830 electroporator (Genetronics, San Diego, CA, USA), which were then incubated at $38^{\circ} \mathrm{C}$ for $72 \mathrm{~h}$ (embryonic day 5 (E5)) in a humidified chamber. Electroporated samples were checked for green fluorescence protein (GFP) expression upon tissue collection; only tissue containing $\mathrm{GFP}^{+}$cells was further analyzed.

\section{Immunohistochemistry}

Embryos were fixed in $4 \%$ paraformaldehyde for $2-4 \mathrm{~h}$ at $4{ }^{\circ} \mathrm{C}$ and paraffin embedded. Immunofluorescence and confocal image analyses were performed on paraffin sections as described previously (Matsuoka et al. 2003). The primary antibodies used were rabbit $\boldsymbol{\alpha}$-mouse MafB (1:5000), rabbit $\boldsymbol{\alpha}$-mouse MafA (1:1000), rabbit $\boldsymbol{\alpha}$-cMaf 153 (Santa Cruz Biotechnology), guinea pig $\boldsymbol{\alpha}$-glucagon (1:2000, Linco Research, St Charles, MO, USA), and guinea pig $\alpha$-insulin (1:2000, Linco Research), with secondary biotin, $\mathrm{Cy} 3-$, or Cy2-conjugated donkey, $\boldsymbol{\alpha}$-guinea pig, and $\alpha$-rabbit IgG (1:500; Jackson ImmunoResearch, West Grove, PA, USA). Peroxidase staining was performed using the DAB substrate kit (Vector Labs, Burlingame, 
CA, USA). Fluorescent images were captured with a Zeiss LSM 510 confocal microscope using an optical depth of $1 \mu \mathrm{m}$. At least three samples were collected and analyzed for each electroporated construct.

\section{Real-time (RT) PCR}

Total RNA from E5 electroporated chick gut tubes was prepared using the RNeasy kit (Qiagen) and treated with DNaseI using the Versagene RNA DNase kit (Gentra Systems, Minneapolis, MN, USA) to remove genomic DNA. TAQMAN RT-PCR reverse transcript reagents (Applied Biosystems, Foster City, CA, USA) were used to generate cDNAs from $0.5 \mu \mathrm{g}$ RNA. PCRs were performed with SYBR Green Master Mix reagents (Applied Biosystems), and the results analyzed using the Applied Biosystems Prism 7000 sequence detection system and software. The following chicken cDNA primer sets were used in the amplification step: insulin top (5'-ATGTCGAGCAGCCCCTAGTG-3'), bottom (5'-TCGTATTCCTCCTGCTGGAAA-3'); 18S, top $\left(5^{\prime}-\mathrm{AGTCCCTGCCCTTTGTACACA}-3^{\prime}\right)$, bottom $\left(5^{\prime}\right.$-GATCCGAGGACCTCACTAAAC- $\left.3^{\prime}\right)$. A sample point consisted of three biological replicates, with each experiment performed on three separate occasions.

\section{DNA constructs}

Mouse wild-type and chimeric MafA and MafB coding sequences were expressed from the $\mathrm{pCIG}$ vector containing a CMV enhancer, $\beta$-actin promoter, and an internal ribosome entry sequence (IRES) followed by a nuclear localized GFP (Megason \& McMahon 2002). The chimeric MafA and MafB constructs were generated by PCR using standard molecular biology techniques. The amino acid (aa) sequences contained in each were: MafA/B, A: amino acids (aa) 1-209, B: aa 187-323; MafB/A, B: aa 1-188, A: aa 206-359; Maf B/A/A, $\mathrm{B}:$ aa $1-76$, A: aa 72-359; MafA/B/A, A: aa 1-73, B: aa 77-187, A: aa 206-359. The correctness of constructs was verified by DNA sequencing. The mouse pCIG-Pdx1 and pCIG-Ngn3 expression plasmids have been described previously (Grapin- Botton et al. 2001). The glucagon-476 (GLU-476 (Lee et al. 1992)) and insulin II-238 (INS-238 (Zhao et al. 2000)) firefly luciferase reporters contain rat gene sequences from nucleotides $-476 /+10$ and $-238 /+2$ respectively.

\section{Transfection assays}

Monolayer cultures of HeLa cells were maintained as described previously (Matsuoka et al. 2003). INS-238 or GLU-476 were transfected with Maf-pCIG constructs and thymidine kinase-driven renilla LUC (phRL-TK; Promega) using the lipofectamine reagent (Invitrogen). Extracts were prepared 40-48 h later and analyzed for firefly and renilla luciferase activity using the Dual-Luciferase Reporter Assay System (Promega). All of the chimeric MafA/B constructs were also found to synthesize a protein of the expected molecular weight and DNA-binding properties by western and gel shift analyses (data not shown).

\section{Results}

Insulin is induced upon ectopic MafA, but not MafB, expression in developing chick endoderm

To assess whether MafA or MafB expression could induce the production of hormone ${ }^{+}$cells in chick endoderm, mouse MafA and MafB were ectopically introduced into embryos using in ovo electroporation. The conditions used for electroporating the CMV enhancer (CMV)/ $\beta$-actin promoter-driven MafA and $\mathrm{MafB}$ vectors favor ectopic expression in endoderm (Grapin-Botton et al. 2001, Xu et al. 2006). Embryos were electroporated at $\mathrm{HH}$ stage 11-13 and analyzed immunohistochemically for the presence of insulin ${ }^{+}$and glucagon ${ }^{+}$cells after 48 (i.e. when the dorsal and ventral pancreatic buds are first visible) or $72 \mathrm{~h}$ (when a branching epithelial architecture of the pancreas is present).

Mouse MafA and MafB expression was detected in anterior and posterior gut tubes, but not the pancreas anlage (Figs 1 and $2 \mathrm{~B}$ ). Insulin was induced in $\sim 80 \%$ of electroporated $\mathrm{MafA}^{+}$cells, which were organized into small clusters close to the gut epithelium. However, MafA did not activate glucagon or
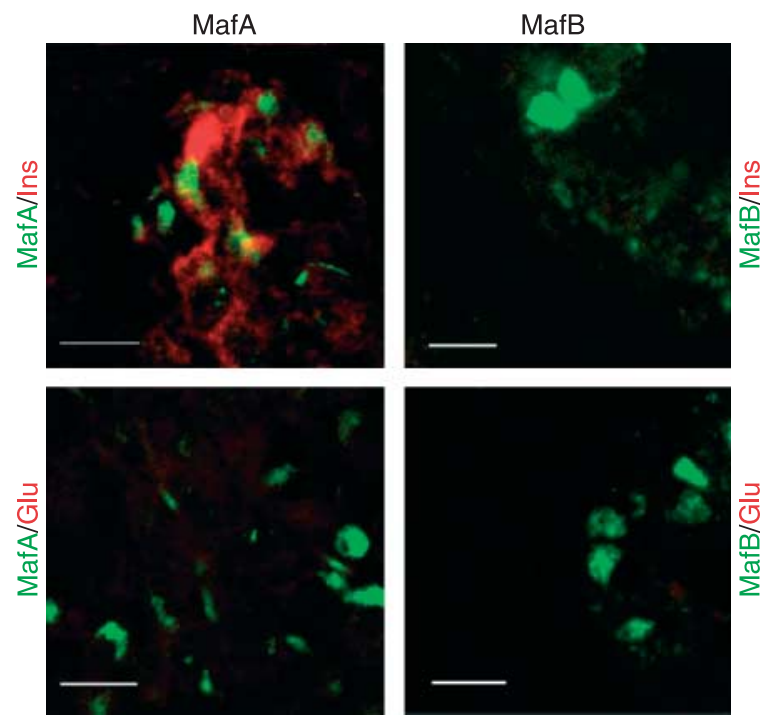

Figure $1 \mathrm{MafA}$, but not $\mathrm{MafB}$, induces insulin expression in chick gut endoderm. Double immunofluorescence staining of E5 chick embryo sections electroporated with MafA or MafB expression plasmids. Sections were stained with $\alpha$-MafA or $\alpha$-MafB (green) and $\alpha$-insulin (Ins) or $\alpha$-glucagon (Glu) (red). Only the mouse $\alpha$-MafA and $\alpha$-MafB antibodies effectively recognize the electroporated mouse proteins, although MafA is produced in the chicken pancreas (Lecoin et al. 2004). MafA ${ }^{+}$cells expressed insulin, whereas no hormone production was detected in $\mathrm{MafB}^{+}$cells. Insulin and MafA were co-expressed in $\sim 80 \%$ of the $\mathrm{MafA}^{+}$cells (230 out of 284 cells). Scale bar $=10 \mu \mathrm{M}$. 
A

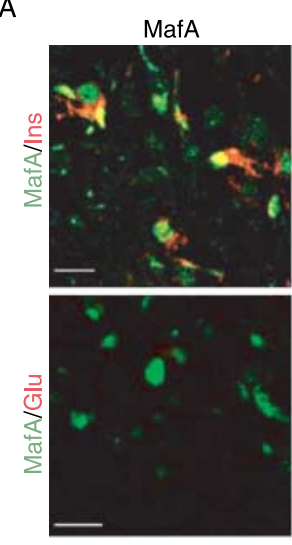

D

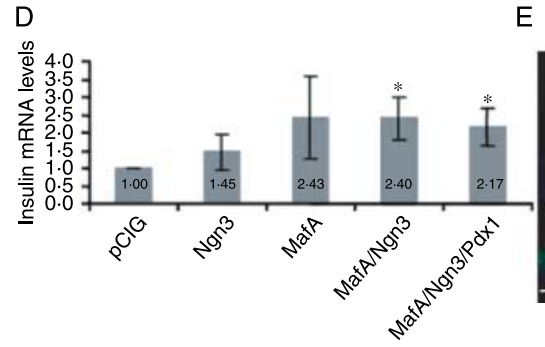

MafA+Ngn3
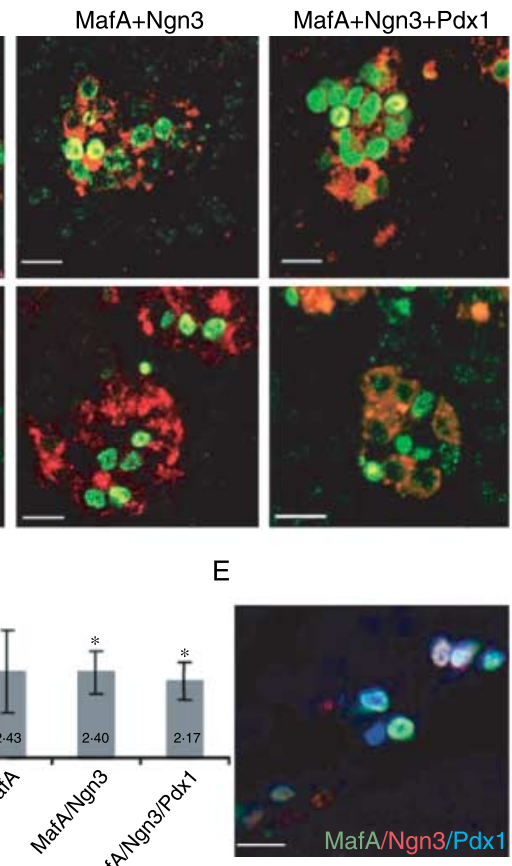

B

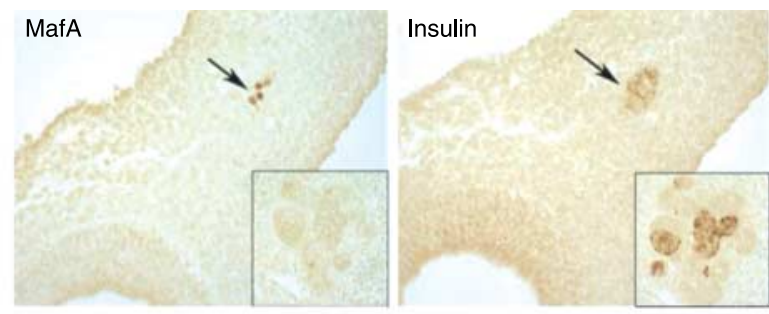

C

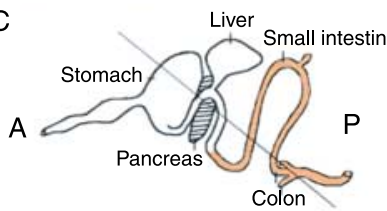

$\mathrm{F}$

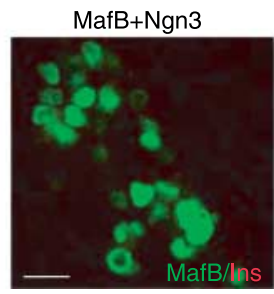

MafB+Ngn3

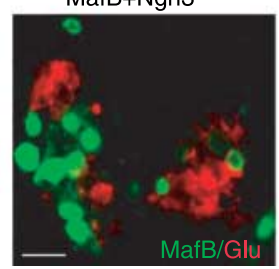

Figure 2 Co-expression of MafA with Ngn3 and/or Pdx1 produces hormone ${ }^{+}$cell clusters. (A) Double immunofluorescence staining of E5 chick embryo sections electroporated with MafA, Ngn3, and/or Pdx1 expression plasmids, as indicated. MafA (green) was detected only in insulin ${ }^{+}$cells (red), and not in glucagon ${ }^{+}$cells (red). MafA was present in 95\% (152 out of 159 cells) and 93\% (91 out of 98 cells) of insulin ${ }^{+}$cells in MafA+ Ngn3 - or MafA + Ngn3 + Pdx1-treated cells respectively. (B) Peroxidase staining for MafA and insulin (brown) in E5 chick embryo sections electroporated with MafA + Ngn3 + Pdx1. Mouse MafA was not detected in the pancreas anlage (insert); as expected, insulin was present in both. (C) Schematic of the chick digestive system. Electroporated cells were detected only in the anterior and posterior gut tubes (orange). The black line indicates the plane of section shown in (B). (D) MafA electroporation induces Insulin mRNA expression in E5 chick endoderm. All of the expression data were derived by real-time PCR analysis and are expressed as the normalized fold difference relative to the pCIG vector alone. ${ }^{*} P<0 \cdot 05$, as determined by ANOVA analysis. The values shown represent mean \pm s.D. (E) Triple immunofluorescence staining of E5 chick embryo sections electroporated with MafA + Ngn3 + Pdx1. MafA (green), Pdx1 (blue), and Ngn3 (red) are co-expressed in electroporated cells. (F) Double immunofluorescence staining of E5 chick embryo sections electroporated with MafB and Ngn3 expression plasmids. Co-expression of MafB and Ngn3 does not induce insulin (red) expression, while glucagon ${ }^{+}$cells (red) were detected. Scale bar $=10 \mu \mathrm{M}$.

a number of transcriptional regulators associated with pancreas development in in situ hybridization or immunohistochemistry assays, including Nkx2.2, Is11, NeuroD1, Pdx1, and Pax6 (data not shown). Furthermore, MafB did not induce glucagon, insulin, or any other analyzed islet-enriched product (Figs 1 and 2F; data not shown). These results suggest that there are unique features of MafA versus MafB, which allow Insulin activation, although not a more general endocrine differentiation program in this chick assay system.

\section{Expression of MafA and Ngn3 results in the formation of insulin- and glucagon-producing islet-like cell clusters}

Next, we examined whether co-electroporation of MafA and MafB with other regulators of $\beta$-cell differentiation could augment insulin induction and islet formation. Previous studies have shown that Ngn3 induced the differentiation of glucagonand somatostatin-producing cells upon in ovo electroporation of chick gut endoderm (Grapin-Botton et al. 2001). Furthermore, the expression of $\mathrm{Ngn} 3$ promotes the activation of endogenous
Pax6, Is11, Hlxb9, NeuroD, and Nkx2.2 transcription factor expression. However, these key islet regulators were unable to activate islet hormone production upon over-expression in chick, although Pdx1 caused the formation of ectopic bud structures similar to that observed during pancreatic development (Grapin-Botton et al. 2001).

Co-expression of Ngn3 and MafA led to the formation of islet-like structures, with insulin-producing cells located in the core and the more plentiful Ngn3-induced glucagon ${ }^{+}$cells on the periphery (Fig. 2A). Ngn3 alone induces glucagon ${ }^{+}$cell formation, but not insulin ${ }^{+}$(Supplementary Fig. 1, which can be viewed online at http://joe.endocrinology-journals.org/ content/vol198/issue2/; Grapin-Botton et al. 2001). There was no apparent change in insulin ${ }^{+}$cell number upon further addition of $\mathrm{Pdx} 1$, or after $\mathrm{Pdx} 1+$ MafA treatment (Fig. 2A). In addition, MafB had no effect on insulin ${ }^{+}$and glucagon ${ }^{+}$ cell number when co-electroporated with Ngn3 (Fig. 2F). Co-electroporation of MafA with MafB also did not result in the production of more insulin-producing cells than with MafA alone (Supplementary Fig. 1). A 2-4-fold induction of Insulin 
mRNA in MafA + Ngn3 - and MafA + Ngn3 + Pdx1treated samples was observed over vector or Ngn3-electroporated cells (Fig. 2D), which is significant considering the small percentage of insulin ${ }^{+}$cells produced by electroporation. The absence of other cooperating factors normally present in developing $\beta$-cells presumably prevented higher level Insulin expression. Notably, the insulin ${ }^{+}$and glucagon ${ }^{+}$cells detected were in a region of endoderm distinct from the developing pancreas (Fig. 2B and C).

The MafA DNA-binding/dimerization domain is a major determinant of insulin induction

MafA/MafB chimeric proteins were generated to identify which region(s) of MafA are critical for Insulin induction. The level of identity between MafA and MafB is the greatest within their N-terminal activation and C-terminal DNA-binding/ dimerization domains (Fig. 3). MafA and MafB activity can be assayed in a very sensitive manner in HeLa cell lines, as both Insulin- and Glucagon-driven reporter constructs are activated to high levels in transient transfection assays (Matsuoka et al. 2003, Artner et al. 2006, Nishimura et al. 2006). By contrast, little to no change in reporter activity is observed in MafA- and MafBtransfected $\alpha$ - or $\beta$-cell lines (data not shown).

Each of the MafA/B chimeric constructs was capable of independently activating the transfected hormone-driven reporter constructs in HeLa cells (Fig. 3B), a result expected considering that MafA and MafB have similar transactivation and protein stability properties (Matsuoka et al. 2003, Nishizawa et al. 2003, Zhao et al. 2005). The subtle difference in relative activation of the $\mathrm{N}$-terminal spanning MafA/B constructs likely reflects their activation domain strength, as determined in mammalian cell transfection experiments ((Nishizawa et al. 2003), Zhao \& Stein unpublished).

The most active insulin-inducing chimeric construct was $\mathrm{MafB} / \mathrm{A} / \mathrm{A}$ in the in ovo assay, which contains the $\mathrm{N}$-terminal aa 1-77 transactivation domain of MafB fused to aa $77-351$ of the hinge and DNA-binding region of MafA (Fig. 4). Like wild-type MafA, the activity of MafB/A/A was enhanced in the presence of Ngn3. By contrast, a larger N-terminal (aa 1187) MafB region fusion, $\mathrm{MafB} / \mathrm{A}$, only induced endogenous insulin expression in the presence of Ngn3. The same property was found with $\mathrm{MafA} / \mathrm{B} / \mathrm{A}$, wherein only the hinge-spanning region of MafA was exchanged (i.e. aa 77187). Significantly, MafA/B, which contains the MafB DNAbinding/dimerization domain, was inactive alone or in the presence of Ngn3 (Fig. 4), like MafB (Fig. 2F). As expected none of the chimeric Maf proteins were capable of influencing glucagon cell production (Supplementary Fig. 2, which can be viewed online at http://joe.endocrinology-journals.org/content/vol198/issue2/). However, both MafA/B and MafB activated hormone-driven expression in HeLa cells (Fig. 3). Together, these results suggest that the MafA DNA-binding/leucine-zipper dimerization domain is indispensable for Insulin activation in the in ovo assay, while the activation domain of either MafA or MafB was functional.
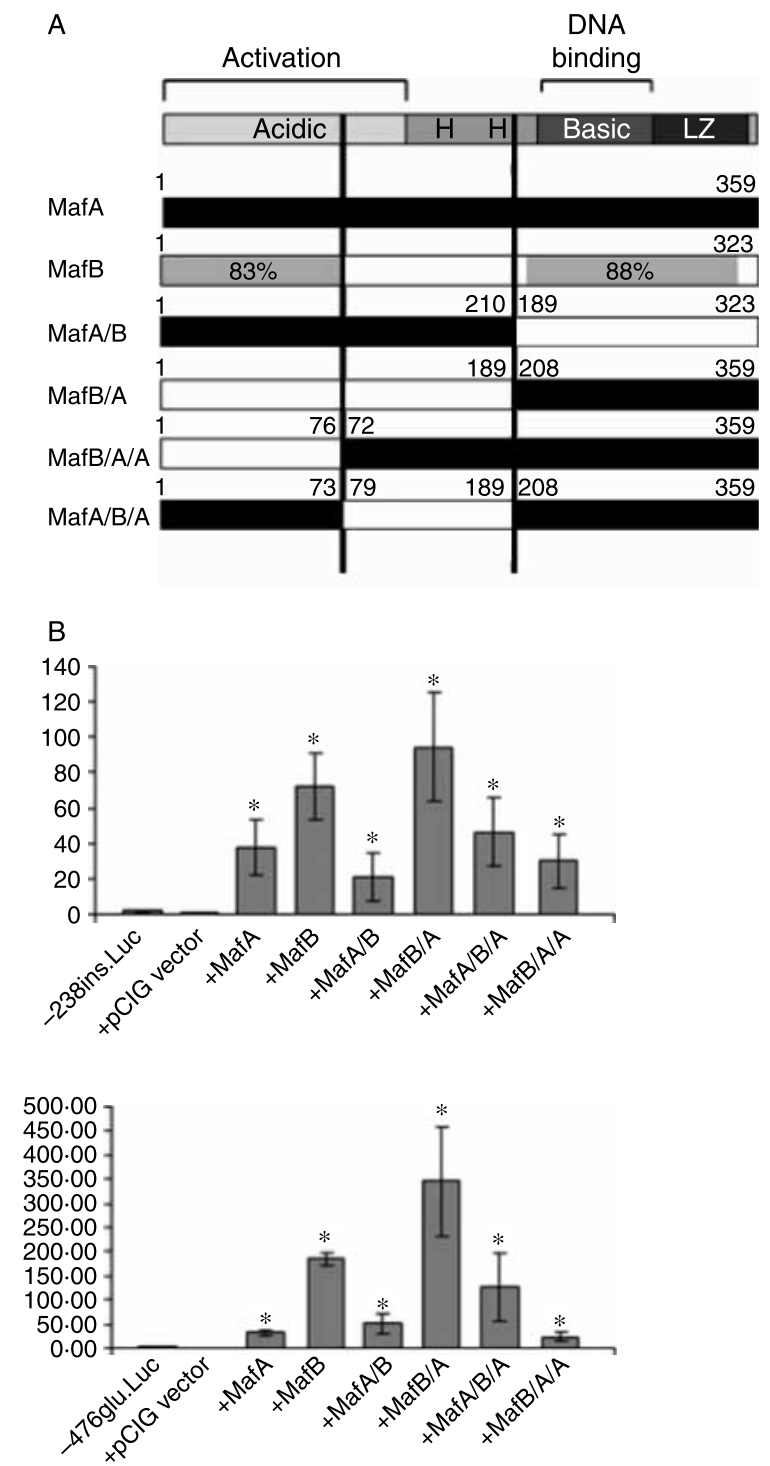

Figure 3 Each of the MafA/MafB chimeras can activate Insulin- and Glucagon-driven expression in cell lines. (A) Schematic of MafA, $\mathrm{MafB}$, and the chimeric proteins. The sequence identity within the conserved activation (aa 1-75) and DNA-binding/dimerization regions is shown. (B) The ability of MafA, MafB, and the chimeric proteins to activate Glucagon- and Insulin-driven reporter constructs in HeLa cells was compared. The chimeric constructs were capable of activating Insulin- and Glucagon-driven reporter constructs. Normalized luciferase activities \pm s.D. are presented relative to the $p C I G$ vector alone $(n=3) .{ }^{*} P<0 \cdot 05$.

MafA and MafB bind within the $5^{\prime}$ promoter region of Insulin and Glucagon in mouse endocrine islet cells

Since MafA specifically activates endogenous Insulin hormone expression in chick embryos and mammalian pancreatic cell lines (Matsuoka et al. 2004, 2007), we next analyzed whether this resulted from differentially binding to Insulin and Glucagon transcriptional regulatory sequences. MafB is expressed 

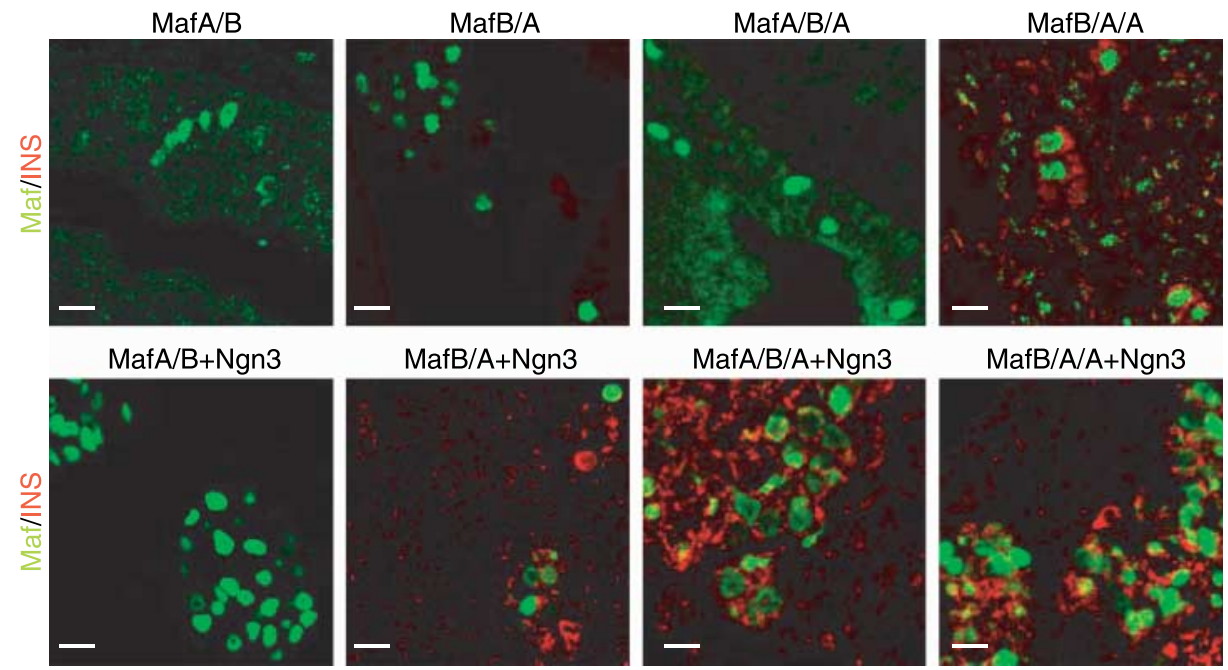

Figure 4 The C-terminal DNA-binding/dimerization domain of MafA is critical to Insulin activation. Double immunofluorescence analysis of chimeric Maf proteins. E5 chick embryo sections represent electroporations with chimeric Maf proteins alone (top) or in the presence of Ngn3 (bottom). Sections were stained with $\alpha$-Maf (green) and $\alpha$-insulin (red). Insulin expression was induced most effectively with MafB/A/A, with low-level expression detected from MafB/A and MafA/B/A in the presence of Ngn3. Scale bar $=10 \mu \mathrm{M}$.

essentially in all insulin ${ }^{+}$and glucagon ${ }^{+}$cells in the E18.5 mouse pancreas (Artner et al. 2006, Nishimura et al. 2006), while MafA is only in insulin ${ }^{+}$cells (Matsuoka et al. 2004). As expected, MafB was bound to the Insulin and Glucagon control regions in ChIP assay (Artner et al. 2006, 2007). Strikingly, $\alpha$-MafA antibodies also precipitated these hormone control regions in E18.5 pancreata, although chromatin treated with normal rabbit IgG did not (Fig. 5A). In addition, MafA antibodies did not precipitate control sequences from the $P E P C K$ gene, which is not expressed in islet endocrine cells. MafA was also capable of selectively binding to Insulin and Glucagon control sequences in $\beta$ TC3 cells, an insulin ${ }^{+} \beta$-cell line that produces MafA and not MafB ((Matsuoka et al. 2003), Fig. 5B), while MafB occupied the same hormone transcriptional control region in an $\alpha$-cell line, $\alpha$ TC6.

The ChIP data demonstrate that MafA and MafB are bound to the Insulin and Glucagon promoters during development and likely in adult islets. Binding is indicative of positive control in developing $\alpha$ - and $\beta$-cells, but may also suggest an involvement in negative regulation during this time and in adults. Significantly, MafA was recently shown to act as both an activator and repressor of CHOP-10/GADD153 transcription in $\beta$-cells (Lawrence et al. 2007).

\section{Discussion}

Previous studies have established that MafA and MafB are critical to $\beta$-cell function (Zhang et al. 2005) and $\alpha$ - and $\beta$-cell differentiation (Artner et al. 2007, Nishimura et al. 2008) respectively. To assess whether MafA and MafB were both sufficient to initiate the differentiation of hormone-producing cells, we performed in ovo electroporation experiments in developing chick endoderm. MafA was uniquely capable of inducing Insulin expression in this model system, whereas MafB did not activate either Insulin or Glucagon transcription nor could a variety of other key pancreatic transcription factors, including Pdx1, Isl1, Pax6, Pax4, Hb9, and MNR2 (Fig. 2; (GrapinBotton et al. 2001)). On the other hand, such distinct functional dissimilarities were not revealed between MafA and MafB in transient co-transfection assays with Insulin-, Glucagon-, or lens Crystallin-driven reporters (Yoshida \& Yasuda 2002, Matsuoka et al. 2003, Nishizawa et al. 2003, Nishimura et al. 2006), cell transformation experiments (Nishizawa et al. 2003), or in vitro gel shift assays (Matsuoka et al. 2003).

The inability of MafB to stimulate hormone expression in the in ovo electroporation assay implies that cooperating factors mediating activation during mouse pancreatic development are lacking in chick endoderm. Certain key Insulin stimulatory factors must also be absent in mammalian $\alpha$-cells (e.g. $\alpha$ TC6 cells), precluding activation even while bound to endogenous control sequences (Fig. 5). The more limited activity of MafB was expected, since its relatively broad expression pattern in other endodermal tissues, including cells in the villi of the duodenum (Artner et al. 2006), does not support a master regulatory function. By contrast, MafA is unusual in being exclusively expressed in insulin $^{+}$cells within the pancreas, consistent with the observations supported here of a fundamental role in promoting Insulin transcription. We believe that both MafA and $\mathrm{MafB}$ are critical to mammalian $\beta$-cell maturation, with MafB acting during development and MafA principally after birth. The data from MafA (Zhang et al. 2005) and MafB (Artner et al. 2007, Nishimura et al. 2008) mutant mice 
A
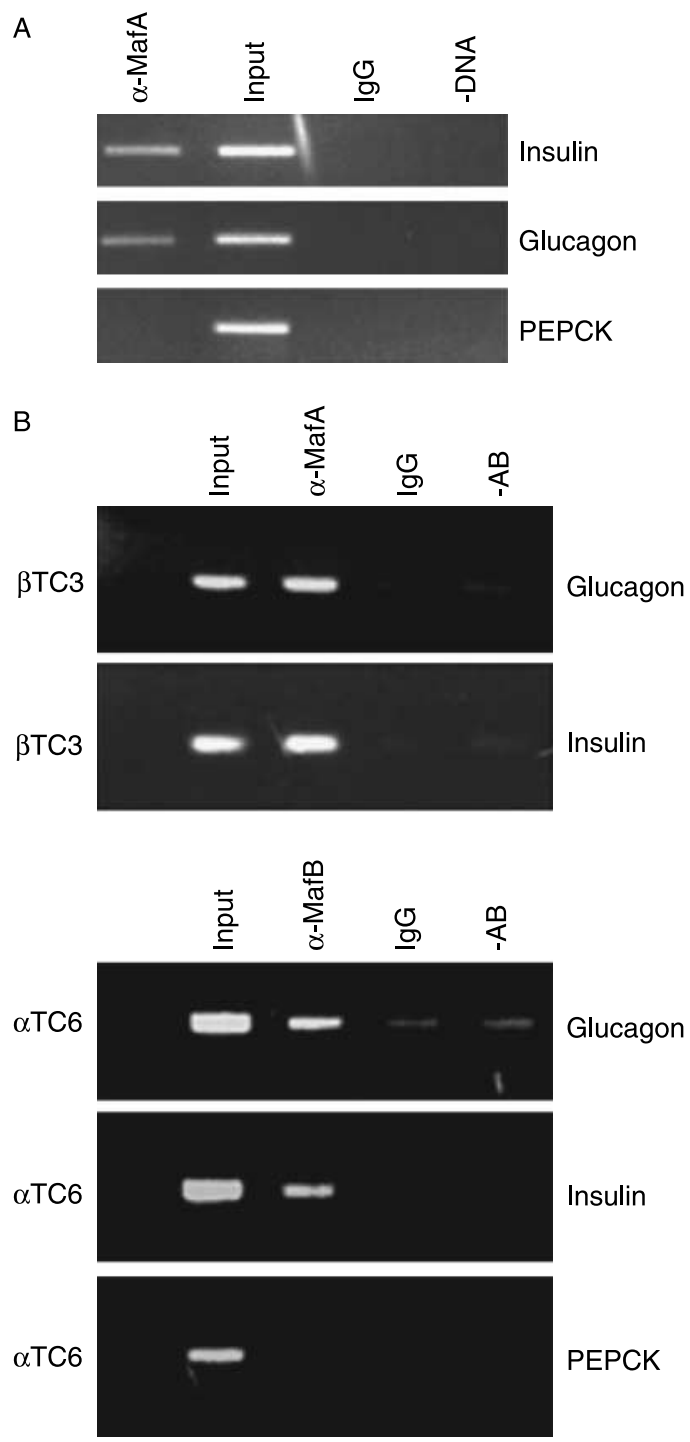

Figure $5 \mathrm{MafA}$ and MafB bind to the Insulin and Glucagon promoter region in vivo. Formaldehyde-cross-linked chromatin from (A) E18.5 pancreatic and (B) $\beta$ TC3 and $\alpha$ TC 6 cells was incubated with antibodies raised to MafA or MafB, as indicated. Immunoprecipitated DNA was analyzed by PCR with primers specific to transcriptional regulatory sequences of mouse Insulin, Glucagon, and PEPCK. As controls, PCR was performed with input DNA (1:100 dilution), DNA precipitated by normal rabbit immune serum, and DNA that was precipitated in the absence of antiserum.

support this proposal as well as our findings showing that MafB is up-regulated at E18.5 in our $\beta$-cell-specific MafA knockout mice (Artner \& Stein unpublished results).

Ngn3 is critical to the formation of all mammalian endocrine islet cell types (Gradwohl et al. 2000). Ectopic expression of this basic helix-loop-helix transcription factor also led to the production of glucagon- and somatostatinproducing cells, but not insulin, in chick embryonic endoderm (Grapin-Botton et al. 2001). MafA co-expression studies with Ngn3 and Pdx1 were performed to determine whether a more mature insulin-producing cell type would be made, since cooperation between transcription factors is required for $\beta$-cell differentiation. Islet-like clusters of insulin $^{+}$and glucagon ${ }^{+}$cells were produced when MafA was expressed with Ngn3 or Ngn3 + Pdx1 (Fig. 2). The amount of insulin in these cells also appeared greater than in those produced by MafA alone, as judged by the assessment of the immunohistochemical data. However, no quantitative difference in Insulin mRNA was found between MafA alone and MafA + Ngn3-electroporated cells. As a consequence, the apparent increase in insulin protein reflects a posttranscriptional process, with translation reflecting the primary means of increasing insulin levels in mammalian $\beta$-cells (Itoh \& Okamoto 1980, Welsh et al. 1986). MafA was also detected only in insulin ${ }^{+}$cells (i.e., roughly 95\% of MafA cells express insulin), implying that the specific activation properties of this factor were not altered upon co-expression with the Ngn3and Pdx1-potentiating factors (Fig. 2E).

MafA/MafB chimeric proteins were developed to localize the region(s) within MafA required for activation of Insulin transcription in the in ovo electroporation assay. The DNAbinding and leucine-zipper/dimerization domain spanning region of MafA appeared to be indispensable, as only the MafA/B chimera and MafB were completely inactive (Figs 1, 2 and 5). Unfortunately there is no obvious structural or sequence motif unique to MafA within this highly conserved region. By contrast, each of the chimeras was capable of effectively activating transfected Insulin- and Glucagon-driven reporters in HeLa cells. Sequences within the N-terminal transactivation domain region of MafA may also selectively contribute to in ovo activation, since MafA was more active than the MafB/A/A chimera. Although we cannot rule out that protein stability contributes to this difference, it is noteworthy that distinguishing activation domain functions have been observed in other systems (Yoshida \& Yasuda 2002, Nishizawa et al. 2003).

The inability of MafA (or MafB) to activate endogenous Glucagon (or Insulin) did not appear to reflect differential binding, as both large Maf factors were capable of specifically binding and stimulating Insulin- and Glucagon-driven reporter expression in gel shift and transient transfection assays (Matsuoka et al. 2003, Artner et al. 2006, Nishimura et al. 2006). However, these in vitro experiments do not account for the native chromatin structure of endogenous genes, which may preclude physiological regulation. As a consequence, ChIP assays were performed to determine whether MafA and MafB physically associated with endogenous Insulin and Glucagon control sequences. We observed occupancy of Insulin and Glucagon $5^{\prime}$-flanking control region sequences in both $\beta$ TC3 and $\alpha$ TC6 cells by MafA and MafB (Fig. 5). MafA binding to Glucagon in E18.5 pancreata demonstrated that occupancy was also observed in developing $\beta$-cells in vivo. Although the physiological significance of this observation is unclear, it suggests that MafA may act as a repressor of Glucagon transcription in $\beta$-cells, an activity associated with 
Pdx1 (Ritz-Laser et al. 2003) and recently for MafA in the context of the CHOP-10/GADD153 gene (Lawrence et al. 2007). Repression of glucagon transcription by $\mathrm{Pdx} 1$ is mediated by the recruitment of Hdac-1 and Hdac-2 (Mosley \& Ozcan 2004). Importantly, these results demonstrate that MafA and MafB directly bind to the Insulin and Glucagon promoter, but that activation is context dependent.

In conclusion, many investigators are working to generate functional insulin-producing $\beta$-like cells from various stem and adult progenitor sources. Although some success has been obtained in generating immature islet-like cells, these appear to be of limited functional value (Segev et al. 2004). The collective results demonstrate a fundamental role for MafA in Insulin gene expression, suggesting that its presence will be essential for generating functional $\beta$-cells for therapeutic transplantation.

\section{Declaration of Interest}

The authors declare no conflict of interest.

\section{Funding}

This work was supported by grants from the National Institutes of Health (P01 DK42502 to R S, 1RO1 DK065949-01A1 to G G, Vanderbilt DRTC pilot grant (DK20593 P\&F) to G G), the American Diabetes Association (7-04RA-116) to R S, and the Juvenile Diabetes Research Foundation (Advanced Postdoctoral 10-2006-5 to I A). Partial support was also provided to the Molecular Biology Core Laboratory by the Vanderbilt University Diabetes Research and Training Center (Public Health Service grant P60 DK20593).

\section{Acknowledgements}

We are grateful to Eva Henderson and Amy Schellhaas for providing technical support. Goat $\alpha-\mathrm{Pdx} 1$ antibody was kindly provided by Dr C V E Wright, and guinea pig $\alpha$-Ngn3 by Dr M Sander.

\section{References}

Ahlgren U, Jonsson J \& Edlund H 1996 The morphogenesis of the pancreatic mesenchyme is uncoupled from that of the pancreatic epithelium in IPF1/PDX1-deficient mice. Development 122 1409-1416.

Artner I \& Stein R 2008 Transcriptional regulation of insulin expression. In Pancreatic Beta Cell in Health and Disease, Ed. GI Bell. Tokyo: Springer Verlag.

Artner I, Le Lay J, Hang Y, Elghazi L, Schisler JC, Henderson E, Sosa-Pineda B \& Stein R 2006 MafB: an activator of the glucagon gene expressed in developing islet $\alpha$ - and $\beta$-cells. Diabetes 55 297-304.

Artner I, Blanchi B, Raum JC, Guo M, Kaneko T, Cordes S, Sieweke M \& Stein R 2007 MafB is required for islet beta cell maturation. PNAS 104 3853-3858.

Ashery-Padan R, Zhou X, Marquardt T, Herrera P, Toube L, Berry A \& Gruss P 2004 Conditional inactivation of Pax6 in the pancreas causes early onset of diabetes. Developmental Biology 269 479-488.

Dasen JS, Liu JP \& Jessell TM 2003 Motor neuron columnar fate imposed by sequential phases of Hox-c activity. Nature 425 926-933.

Del Prato S \& Marchetti P 2004 Beta- and alpha-cell dysfunction in type 2 diabetes. Hormone and Metabolic Research 36 775-781.
Dumonteil E, Laser B, Constant I \& Philippe J 1998 Differential regulation of the glucagon and insulin I gene promoters by the basic helix-loop-helix transcription factors E47 and BETA2. Journal of Biological Chemistry 273 19945-19954.

Gradwohl G, Dierich A, LeMeur M \& Guillemot F 2000 Neurogenin3 is required for the development of the four endocrine cell lineages of the pancreas. PNAS 97 1607-1611.

Grapin-Botton A, Majithia AR \& Melton DA 2001 Key events of pancreas formation are triggered in gut endoderm by ectopic expression of pancreatic regulatory genes. Genes and Development 15 444-454.

Gu G, Wells JM, Dombkowski D, Preffer F, Aronow B \& Melton DA 2004 Global expression analysis of gene regulatory pathways during endocrine pancreatic development. Development 131 165-179.

Hamburger V \& Hamilton HL 1951 A series of normal stages in the development of the chick embryo. Journal of Morphology 88 49-92.

Hussain MA, Miller CP \& Habener JF 2002 Brn-4 transcription factor expression targeted to the early developing mouse pancreas induces ectopic glucagon gene expression in insulin-producing $\beta$ cells. Journal of Biological Chemistry 277 16028-16032.

Itoh N \& Okamoto H 1980 Translational control of proinsulin synthesis by glucose. Nature 283 100-102.

Jonsson J, Carlsson L, Edlund T \& Edlund H 1994 Insulin-promoter-factor 1 is required for pancreas development in mice. Nature 371 606-609.

Lawrence MC, McGlynn K, Naziruddin B, Levy MF \& Cobb MH 2007 Differential regulation of CHOP-10/GADD153 gene expression by MAPK signaling in pancreatic $\beta$-cells. PNAS 104 11518-11525.

Lecoin L, Sii-Felice K, Pouponnot C, Eychene A \& Felder-Schmittbuhl MP 2004 Comparison of maf gene expression patterns during chick embryo development. Gene Expression Patterns 4 35-46.

Lee YC, Asa SL \& Drucker DJ 1992 Glucagon gene 5 '-flanking sequences direct expression of simian virus 40 large $\mathrm{T}$ antigen to the intestine, producing carcinoma of the large bowel in transgenic mice. Journal of Biological Chemistry 267 10705-10708.

Malecki MT, Jhala US, Antonellis A, Fields L, Doria A, Orban T, Saad M, Warram JH, Montminy M \& Krolewski AS 1999 Mutations in NEUROD1 are associated with the development of type 2 diabetes mellitus. Nature Genetics 23 323-328.

Matsuoka TA, Zhao L, Artner I, Jarrett HW, Friedman D, Means A \& Stein R 2003 Members of the large Maf transcription family regulate insulin gene transcription in islet beta cells. Molecular and Cellular Biology 23 6049-6062.

Matsuoka TA, Artner I, Henderson E, Means A, Sander M \& Stein R 2004 The MafA transcription factor appears to be responsible for tissue-specific expression of insulin. PNAS $1012930-2933$.

Matsuoka TA, Kaneto H, Stein R, Miyatsuka T, Kawamori D, Henderson E, Kojima I, Matsuhisa M, Hori M \& Yamasaki Y 2007 MafA regulates expression of genes important to islet beta-cell function. Molecular Endocrinology 21 2764-2774.

Megason SG \& McMahon AP 2002 A mitogen gradient of dorsal midline Wnts organizes growth in the CNS. Development 129 2087-2098.

Melloul D, Marshak S \& Cerasi E 2002 Regulation of insulin gene transcription. Diabetologia 45 309-326.

Mosley AL \& Ozcan S 2004 The pancreatic duodenal homeobox-1 protein $(\mathrm{Pdx}-1)$ interacts with histone deacetylases Hdac-1 and Hdac-2 on low levels of glucose. Journal of Biological Chemistry 279 54241-54247.

Naya FJ, Stellrecht CM \& Tsai MJ 1995 Tissue-specific regulation of the insulin gene by a novel basic helix-loop-helix transcription factor. Genes and Development 9 1009-1019.

Naya FJ, Huang HP, Qiu Y, Mutoh H, DeMayo FJ, Leiter AB \& Tsai MJ 1997 Diabetes, defective pancreatic morphogenesis, and abnormal enteroendocrine differentiation in BETA2/neuroD-deficient mice. Genes and Development 11 2323-2334.

Nishimura W, Kondo T, Salameh T, El Khattabi I, Dodge R, Bonner-Weir S \& Sharma A 2006 A switch from MafB to MafA expression accompanies differentiation to pancreatic beta-cells. Developmental Biology 293 526-539.

Nishimura W, Rowan S, Salameh T, Maas RL, Bonner-Weir S, Sell SM \& Sharma A 2008 Preferential reduction of beta cells derived from Pax6-MafB pathway in MafB deficient mice. Developmental Biology 314 443-456. 
Nishizawa M, Kataoka K \& Vogt PK 2003 MafA has strong cell transforming ability but is a weak transactivator. Oncogene 22 7882-7890.

Offield MF, Jetton TL, Labosky PA, Ray M, Stein RW, Magnuson MA, Hogan BL \& Wright CV 1996 PDX-1 is required for pancreatic outgrowth and differentiation of the rostral duodenum. Development 122 983-995.

Olbrot M, Rud J, Moss LG \& Sharma A 2002 Identification of beta-cellspecific insulin gene transcription factor RIPE3b1 as mammalian MafA. PNAS 99 6737-6742.

Phuc Le P, Friedman JR, Schug J, Brestelli JE, Parker JB, Bochkis IM \& Kaestner KH 2005 Glucocorticoid receptor-dependent gene regulatory networks. PLoS Genetics 1 e16.

Ritz-Laser B, Estreicher A, Klages N, Saule S \& Philippe J 1999 Pax-6 and $\mathrm{Cdx}-2 / 3$ interact to activate glucagon gene expression on the G1 control element. Journal of Biological Chemistry 274 4124-4132.

Ritz-Laser B, Gauthier BR, Estreicher A, Mamin A, Brun T, Ris F, Salmon P, Halban PA, Trono D \& Philippe J 2003 Ectopic expression of the $\beta$-cell specific transcription factor Pdx1 inhibits glucagon gene transcription. Diabetologia 46 810-821.

Sander M \& German MS 1997 The $\beta$ cell transcription factors and development of the pancreas. Journal of Molecular Medicine 75 327-340.

Sander M, Neubuser A, Kalamaras J, Ee HC, Martin GR \& German MS 1997 Genetic analysis reveals that PAX6 is required for normal transcription of pancreatic hormone genes and islet development. Genes and Development 11 1662-1673.

Segev H, Fishman B, Ziskind A, Shulman M \& Itskovitz-Eldor J 2004 Differentiation of human embryonic stem cells into insulin-producing clusters. Stem Cells 22 265-274.

Servitja JM \& Ferrer J 2004 Transcriptional networks controlling pancreatic development and $\beta$ cell function. Diabetologia 47 597-613.

Shapiro AM, Lakey JR, Ryan EA, Korbutt GS, Toth E, Warnock GL, Kneteman NM \& Rajotte RV 2000 Islet transplantation in seven patients with type 1 diabetes mellitus using a glucocorticoid-free immunosuppressive regimen. New England Journal of Medicine 343 230-238.
Stoffers DA, Ferrer J, Clarke WL \& Habener JF 1997 Early-onset type-II diabetes mellitus (MODY4) linked to IPF1. Nature Genetics 17 138-139.

St-Onge L, Sosa-Pineda B, Chowdhury K, Mansouri A \& Gruss P 1997 Pax6 is required for differentiation of glucagon-producing $\alpha$-cells in mouse pancreas. Nature 387 406-409.

Welsh M, Scherberg N, Gilmore R \& Steiner DF 1986 Translational control of insulin biosynthesis. Evidence for regulation of elongation, initiation and signal-recognition-particle-mediated translational arrest by glucose. Biochemical Journal 235 459-467.

Xu Y, Wang S, Zhang J, Zhao A, Stanger BZ \& Gu G 2006 The fringe molecules induce endocrine differentiation in embryonic endoderm by activating cMyt1/cMyt3. Developmental Biology 297 340-349.

Yoshida T \& Yasuda K 2002 Characterization of the chicken L-Maf, MafB and c-Maf in crystallin gene regulation and lens differentiation. Genes to Cells 7 693-706.

Zhang C, Moriguchi T, Kajihara M, Esaki R, Harada A, Shimohata H, Oishi H, Hamada M, Morito N, Hasegawa K et al. 2005 MafA is a key regulator of glucose-stimulated insulin secretion. Molecular and Cellular Biology 25 4969-4976.

Zhao L, Cissell MA, Henderson E, Colbran R \& Stein R 2000 The RIPE3b1 activator of the insulin gene is composed of a protein(s) of approximately $43 \mathrm{kDa}$, whose DNA binding activity is inhibited by protein phosphatase treatment. Journal of Biological Chemistry 275 10532-10537.

Zhao L, Guo M, Matsuoka TA, Hagman DK, Parazzoli SD, Poitout V \& Stein R 2005 The islet $\beta$ cell-enriched MafA activator is a key regulator of insulin gene transcription. Journal of Biological Chemistry 280 11887-11894.

Received in final form 2 May 2008

Accepted 30 May 2008

Made available online as an Accepted Preprint

30 May 2008 